\title{
Danshensu Inhibits $\beta$-Adrenergic Receptors-Mediated Cardiac Fibrosis by ROS/p38 MAPK Axis
}

\author{
Haiyan Lu, ${ }^{b, \#}$ Aiju Tian, ${ }^{a, \#}$ Jimin Wu, ${ }^{a}$ Chengzhi Yang, ${ }^{a}$ Rui Xing, ${ }^{a}$ Pu Jia, ${ }^{b}$ Lingjian Yang, ${ }^{b}$ \\ Youyi Zhang, ${ }^{a}$ Xiaohui Zheng, ${ }^{*}, b$ and Zijian $\mathrm{Li}^{*}, a$ \\ ${ }^{a}$ Institute of Vascular Medicine, Peking University Third Hospital, Key Laboratory of Cardiovascular Molecular \\ Biology and Regulatory Peptides, Ministry of Health, Key Laboratory of Molecular Cardiovascular Sciences, \\ Ministry of Education and Beijing Key Laboratory of Cardiovascular Receptors Research; Beijing 100191, \\ China: and ${ }^{b}$ Key Laboratory of Resource Biology and Biotechnology in Western China, Ministry of Education, \\ Northwest University; Xi'an 710069, China. \\ Received November 27, 2013; accepted March 24, 2014
}

Danshensu, the effective ingredient of the plant Salvia miltiorrhiza (Danshen), has been widely used for treatment of cardiovascular diseases. Cardiac fibrosis is an important process in pathological cardiac remodeling and leads to heart failure. We investigated the effect of Danshensu on $\boldsymbol{\beta}$-adrenergic receptor $(\beta$-AR)-mediated cardiac fibrosis and the involved signaling transduction. Danshensu inhibited cardiofibroblast proliferation and collagen I synthesis induced by isoproterenol (ISO), a selective $\beta$-AR agonist. Phosphorylation of p38 mitogen-activated protein kinase (MAPK), which mediates ISO-induced cardiac fibrosis, was negatively regulated in this process. The negative regulation depended on the ISO inhibition of reactive oxygen species (ROS) production. Taken together, Danshensu may inhibit $\beta$-AR-mediated cardiac fibrosis by negative regulation of ROS-p38 MAPK signaling.

Key words Danshensu; $\beta$-adrenergic receptor; cardiac fibrosis; $\mathrm{p} 38$ MAPK; reactive oxygen specy

Cardiac fibrosis is a pivotal phenomenon in pathological cardiac remodeling and is a hallmark of heart diseases. It is characterized by excessive extracellular matrix accumulation and fibroblast deposition and eventually destroys the organ architecture and abolishes normal function. ${ }^{1-3)}$ Myocardial fibrosis is a major biological determinant of fatal events in cardiac remodeling, including heart failure, severe arrhythmias and sudden cardiac death. ${ }^{4,5}$ Cardiac fibrosis can be caused by various factors, such as the chronic activation of the sympathetic nervous system, myocardial hypoxia, ischemia, senescence, inflammation and hormones. $\beta$-Adrenergic recep- tors ( $\beta$-ARs) and their associated guanine nucleotide regulatory protein ( $\mathrm{G}$ protein)/adenylyl cyclase signal transduction pathways, are central to the regulation of cardiac function. Continuous activation of $\beta$-ARs plays an important role in cardiac dysfunction. ${ }^{6-8)}$ Cardiac function was altered in $\beta_{1}$-AR transgenic mice with cardiac hypertrophy, which could be inhibited by $\beta$-AR blockers. ${ }^{8}$ Consecutive administration with isoproterenol (ISO), a $\beta$-AR agonist, caused cardiac fibrosis in rats. 9,10$)$

p38 mitogen-activated protein kinase (p38 MAPK) and reactive oxygen species (ROS) play a critical role in cardiac
A
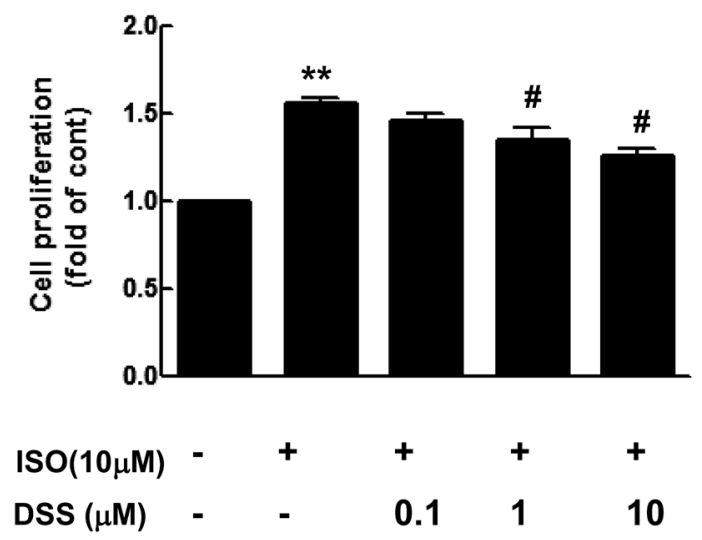

B

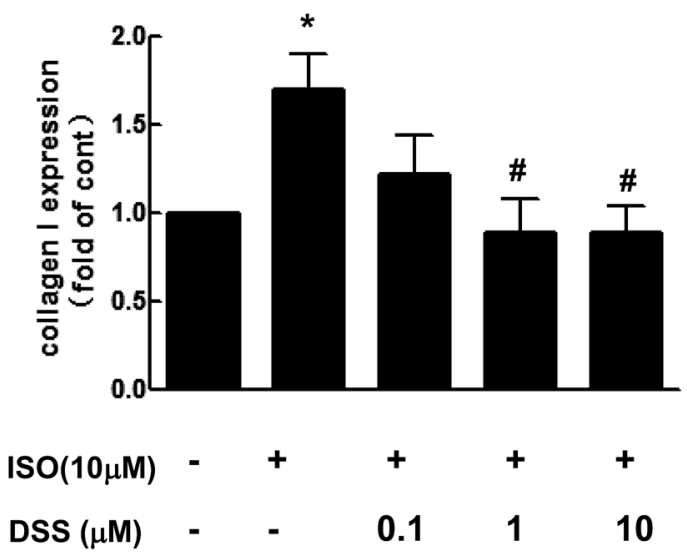

Fig. 1. Effect of Danshensu (DSS) on Isoproterenol (ISO)-Induced Cardiofibroblast (CF) Proliferation and Type I Collagen Synthesis

CFs were treated with vehicle, ISO or DSS plus ISO for $24 \mathrm{~h}$. (A) Cell proliferation was examined by use of the cell counting kit-8 (CCK-8). $* * p<0.01 v s$. vehicle, ${ }^{\#} p<0.05$ vs. ISO. (B) ELISA of type I collagen synthesis. ${ }^{*} p<0.05$ vs. vehicle; ${ }^{\sharp} p<0.05$ vs. ISO. $N=4$.

The authors declare no conflict of interest.

\# These authors contributed equally to this work. 
fibrosis. ROS can activate matrix metalloproteinases, which mediate cardiac fibrosis. ${ }^{11)}$ The $\beta$-adrenergic system may contribute to cardiac dysfunction and extracellular matrix remodelling by activating the $\beta_{2}$-AR-Nox-ROS-p38 MAPK axis. ${ }^{12)}$ Our previous study also found that $\beta$-AR stimulated interleukin 6 production, which is important for activating $\mathrm{p} 38$ MAPK signaling and the consequent cardiac fibrosis. ${ }^{13)}$ Therefore, the axis is a promising target for drugs against cardiac fibrosis.

Danshensu (DSS) is the major water-soluble ingredient extracted from the plant Salvia miltiorrhizae (Danshen). S. miltiorrhizae is used in traditional Chinese medicine and is widely used to treat various microcirculatory disturbancerelated diseases such as cardiovascular disease, cerebrovascular disease, renal dysfunction and liver fibrosis. DSS has biological activities in improving microcirculation, suppressing the formation of ROS, protecting the myocardium against ischemia, ${ }^{14)}$ protecting endothelial cells against injury induced by inflammation, and enhancing anti-apoptosis. ${ }^{15,16)}$ However, the role of DSS in cardiac fibroblasts (CFs) remains unclear. In this study, we assessed the effect of DSS on CFs and its mechanism.

\section{MATERIALS AND METHODS}

Animals One to 2 d-old Sprague-Dawley rats were supplied by the animal research center of Beijing Medical University. The experiments were performed in accordance with the U.S. National Institutes of Health Guidelines for the Use of Laboratory Animals and were approved by the Beijing Medical University Committee on Animal Care. Neonatal rat CFs were isolated as described previously. ${ }^{17)}$

CF Culture Neonatal CFs were isolated from minced rat ventricles digested with $0.01 \%$ collagenase II (Worthington, Columbia, NJ, U.S.A.). Cells were collected and plated for $2 \mathrm{~h}$ at $37^{\circ} \mathrm{C}$. Unattached cardiomyocytes were removed. CFs were cultured in Dulbecco's modified Eagle's medium (DMEM) (Hyclone, Beijing) with 10\% fetal bovine serum (Hyclone Laboratories, Omaha, NE, U.S.A.) at $37^{\circ} \mathrm{C}{ }^{18)}$

CF Proliferation Assay CFs were counted by use of the cell counting kit-8 (CCK-8) (Dojindo, Japan). ${ }^{19)} \mathrm{CFs}$ at passage 2 or 3 were digested with $0.25 \%$ trypsin and ethylene diamine tetraacetic acid (EDTA), then cultured in 96-well

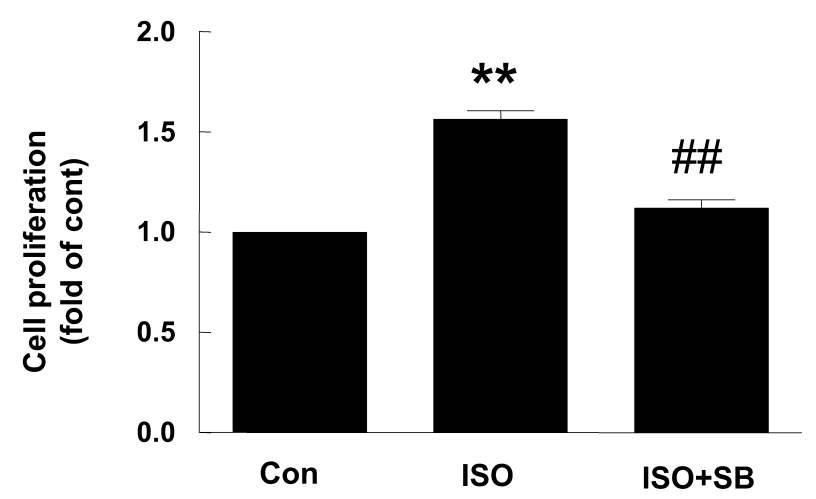

Fig. 2. Effect of p38 Inhibitor SB202190 on CF Proliferation Induced by ISO

CFs were treated with vehicle, ISO or ISO plus SB202190 (SB) for $24 \mathrm{~h}$ $* * p<0.01$ vs. vehicle; ${ }^{\#} p<0.01$ vs. ISO. $N=3$.
A

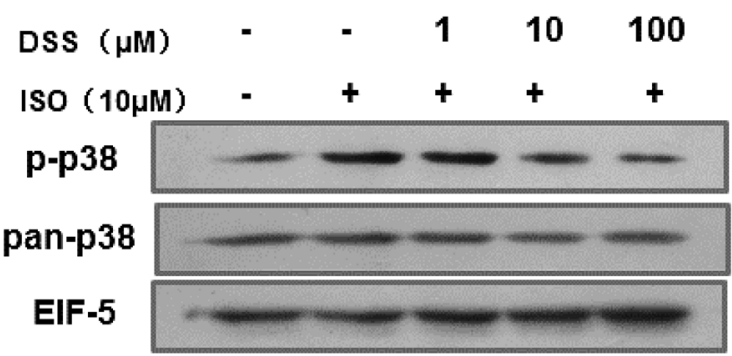

B

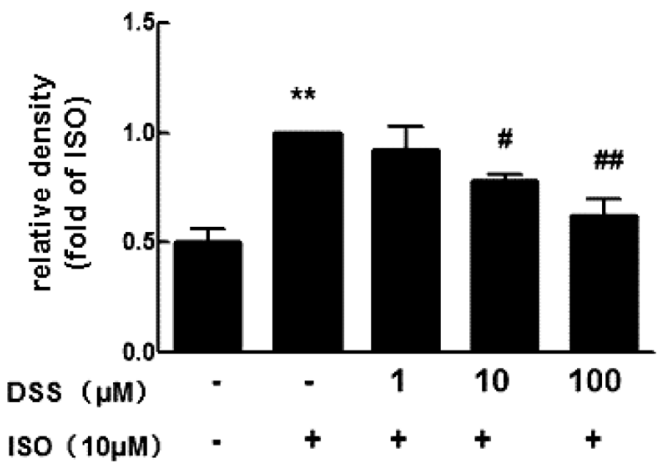

Fig. 3. Effect of DSS on ISO-Induced p38 Mitogen-Activated Protein Kinase (MAPK) Activation

CFs were treated with vehicle, ISO or ISO plus DSS for $24 \mathrm{~h}$. (A) Western blot analysis and (B) quantification of p38 MAPK phosphorylation. $* * p<0.01 v s$. vehicle; ${ }^{\#} p<0.05$ vs. ISO; ${ }^{\#} p<0.01$ vs. ISO. $N=3$.
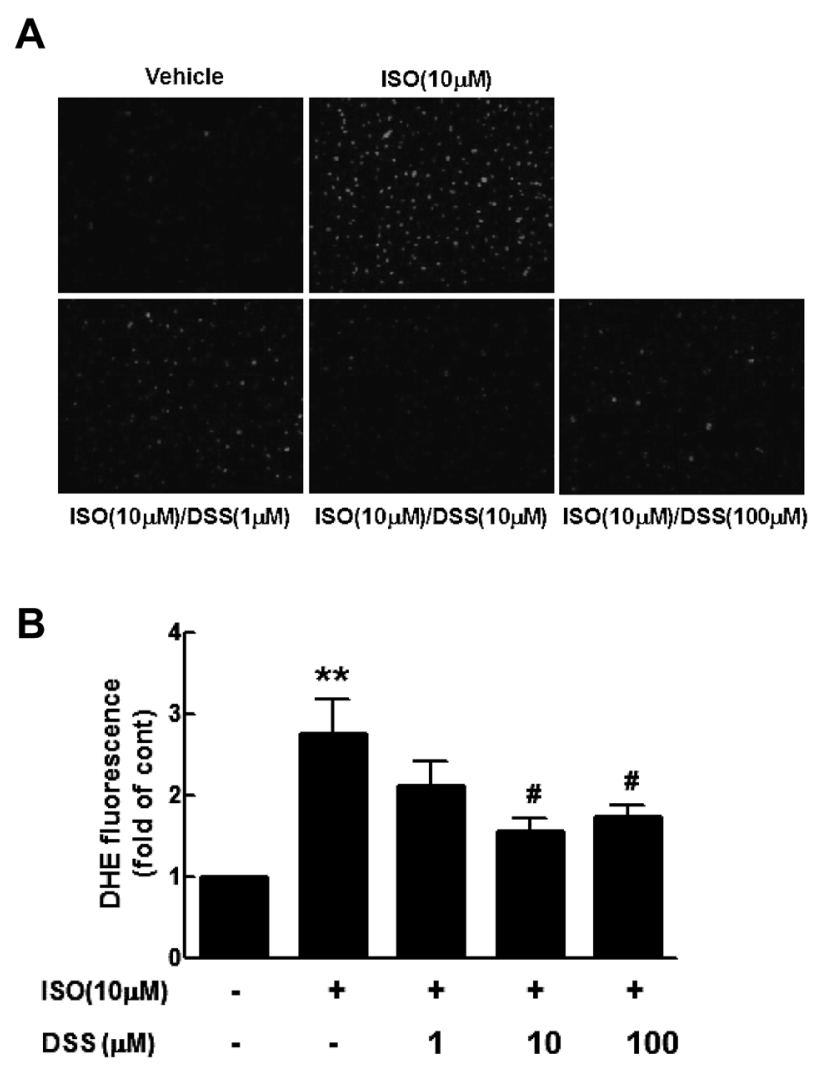

Fig. 4. Effect of DSS on ISO-Induced ROS Production

CFs were treated with vehicle, ISO or ISO plus DSS for $30 \mathrm{~min}$. Intracellular ROS accumulation was assessed with dihydroethidium incubation. (A) Representative micrographs of ROS accumulation. (B) Quantification of intracellular ROS as a ratio to vehicle levels. ${ }^{* *} p<0.01 v s$. vehicle; ${ }^{\sharp} p<0.05 v$ s. ISO. $N=3$. 
plates $\left(3 \times 10^{3}\right.$ to $5 \times 10^{3}$ cells per well $)$ in serum-free, highglucose DMEM with or without concentrations of DSS (The National Institute for Food and Drug Control, China) for $1 \mathrm{~h}$ before treatment with ISO (Sigma). After incubation for $24 \mathrm{~h}$, the supernatant was removed, and $100 \mu \mathrm{L}$ DMEM containing $10 \mu \mathrm{L}$ CCK-8 was added to each well for another $4 \mathrm{~h}$ at $37^{\circ} \mathrm{C}$. The optical density was read at $450 \mathrm{~nm}$ (Microplate Reader Model 550, Bio-Rad).

Enzyme-Linked Immunosorbent Assay (ELISA) The secretion of collagen type I by CFs was measured by use of a commercially available ELISA kit (R\&D Systems, Minneapolis, MN, U.S.A.). All samples were assayed in triplicate.

Measurement of Intracellular ROS Dihydroethidium (DHE) (Molecular Probes, OR, U.S.A.) was used to assess intracellular ROS formation in cultured $\mathrm{CFs}^{20)}$ Cells were loaded with $10 \mu \mathrm{M} \mathrm{DHE}$ for $30 \mathrm{~min}$ at $37^{\circ} \mathrm{C}$, then washed with Hanks' solution. After treatment with ISO $(10 \mu \mathrm{M})$, cells were washed twice with Hanks' solution. ROS production was analyzed by inverted fluorescence microscopy (Leica).

Western Blot Analysis Protein expression was examined by Western blot analysis as described. ${ }^{21)}$ Proteins were electrophoresed and incubated with the antibodies rabbit antiphospho-p38 MAPK (1:1000), anti-p38 MAPK (1:1000), and anti-eukaryotic initiation factor 5 (anti-EIF5) $(1: 5000$, all Cell Signaling Technology).

Statistical Analysis Data are shown as mean \pm S.D. Oneway ANOVA was used to compare groups, with Tukey's test, by use of GraphPad Prism 5.0 (GraphPad Software, La Jolla, CA, U.S.A.). A $p<0.05$ was considered statistically significant.

\section{RESULTS}

DSS Attenuated ISO-Induced CF Proliferation and Type I Collagen Synthesis To evaluate the effect of DSS on cardiac fibrosis induced by ISO, we detected CF proliferation. CF proliferation was significantly increased after stimulation with ISO $(10 \mu \mathrm{M})$ for $24 \mathrm{~h}$ (Fig. 1A). DSS $(0.1,1,10 \mu \mathrm{M})$ significantly inhibited the ISO-increased CF proliferation dose-dependently. ISO $(10 \mu \mathrm{M})$ significantly increased the expression of type I collagen in cultured CFs (Fig. 1B). The increased collagen synthesis was dose-dependently inhibited by DSS $(0.1,1$, $10 \mu \mathrm{M})$. Thus, DSS may attenuate ISO-induced CF proliferation and collagen synthesis.

p38 MAPK Pathway Mediated ISO-Induced CF Proliferation p38 MAPK plays an important role in CF proliferation. $^{22)}$ ISO $(10 \mu \mathrm{M})$ stimulation significantly induced CF proliferation, and the p38 inhibitor SB202190 $(10 \mu \mathrm{M})$ reversed this process, so the p38 signaling pathway mediated ISO-induced CF proliferation (Fig. 2).

DSS Negatively Regulated ISO-Induced Phosphorylation of p38 MAPK in CFs We hypothesized that ISO may stimulate p38 phosphorylation with $\mathrm{CF}$ proliferation and that DSS inhibits this process. The phosphorylation of p38 was significantly increased with ISO $(10 \mu \mathrm{m})$ treatment in CFs and
A

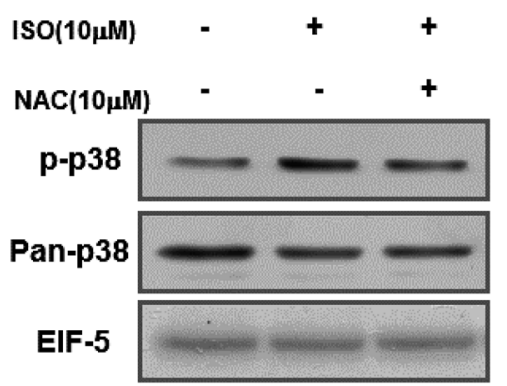

B

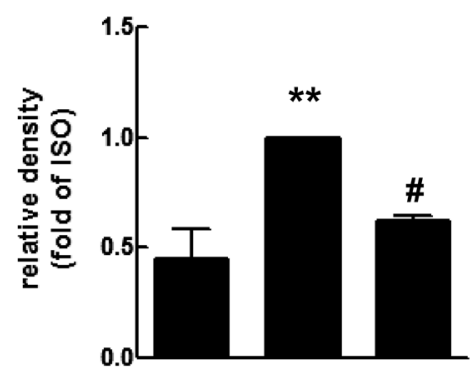

\section{C}

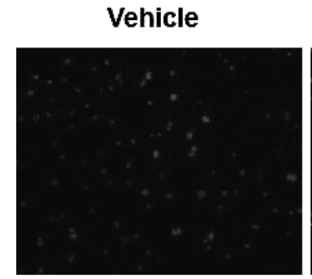
ISO(10 $\mu \mathrm{M})$

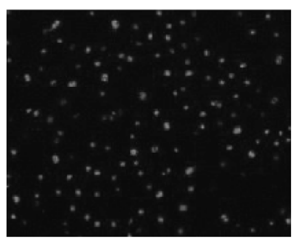
ISO $(10 \mu \mathrm{M}) / \mathrm{SB}(10 \mu \mathrm{M})$

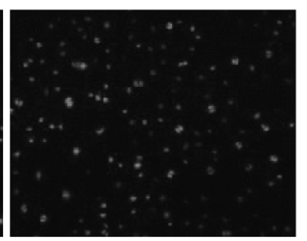

D

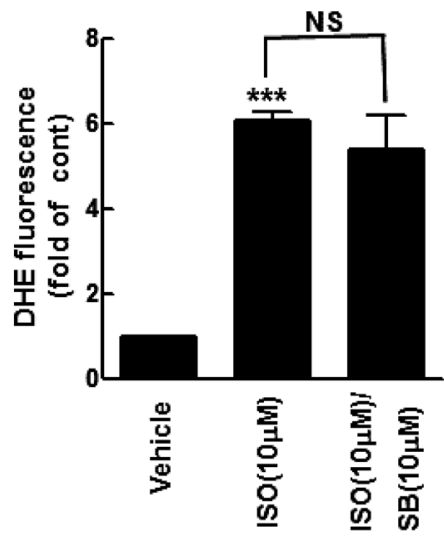

Fig. 5. The Interaction of $\mathrm{p} 38$ MAPK and ROS in CFs Treated with ISO

(A) Western blot analysis and (B) quantification of the effect of scavenging ROS with NAC on p38 MAPK activity. ** $p<0.01 v s$. vehicle; ${ }^{*} p<0.05 v s$. ISO. (C) Representative micrographs and (D) quantification of the effect of $\mathrm{p} 38$ inhibitor SB202190 on ROS activation. $* * * p<0.001$ vs. vehicle; NS: no significance. $N=3$. 
DSS $(1,10,100 \mu \mathrm{M})$ dose-dependently reversed the increased phosphorylation (Fig. 3).

DSS Decreased ISO-Induced ROS Generation in CFs ISO can generate free radicals and stimulate lipid peroxidation, ${ }^{23)}$ and ROS plays an important role in ISO-induced organ fibrosis. ${ }^{24)}$ We found that ISO stimulation for $30 \mathrm{~min}$ significantly promoted ROS production (Fig. 4). DSS (1, 10, $100 \mu \mathrm{M})$ dose-dependently inhibited the increased ROS production. Thus, ISO may trigger an increase in ROS levels in CFs and DSS may reduce the ISO-induced ROS production.

The Phosphorylation of 338 Depended on ISO-Induced ROS Production Previous studies have shown that the ROS-p38 signal pathway is involved in CF proliferation. ${ }^{25)} \mathrm{We}$ found that ROS scavenger (NAC) abrogated the ISO-induced phosphorylation of p38. However, the p38 inhibitor SB202190 did not inhibit ISO-increased ROS production in CFs (Figs. $5 \mathrm{C}, \mathrm{D})$. Thus, increased ROS accumulation mediated the ISOinduced phosphorylation of p38, but p38 activation did not affect ISO-generated ROS in CFs.

DSS Decreased ISO-Induced ROS Production Dependent on Reduced Nicotinamide Adenine Dinucleotide Phos- phate (NADPH) Oxidase 2 (NOX2) Increased ROS accumulation was from imbalance between ROS production and elimination. To clarify the mechanism by which DSS reduces ROS production, we examined the expression of NADPH oxidase and antioxidant enzymes superoxide dismutase (SOD). NADPH oxidase, the major enzymatic source of ROS, was examined in CFs by measuring levels of NOX2 and NOX4, two major NAD(P)H oxidase subunits. The NOX2 protein levels were significantly upregulated in ISO-treated $(10 \mu \mathrm{M}) \mathrm{CFs}$ and reversed by DSS (Fig. 6A). However, either ISO $(10 \mu \mathrm{M})$ or DSS $(10 \mu \mathrm{M})$ treatment had no effect on NOX4 protein expression in CFs (Fig. 6B). Thus, the inhibitory effect of DSS on NADPH oxidase expression was NOX2-dependent. Superoxide dismutase (SOD) is most powerful natural antioxidant enzymes. Here, we determined levels of SOD1 and SOD2, two major forms of SOD, in CFs with treatment. The results showed that both ISO $(10 \mu \mathrm{M})$ and DSS $(10 \mu \mathrm{M})$ treatment had no effect on SOD1 and SOD2 protein expression in CFs (Figs. $6 \mathrm{C}, \mathrm{D})$. Taken together, these results suggested that DSS decreased ISO-induced ROS production depended on NOX2.
A

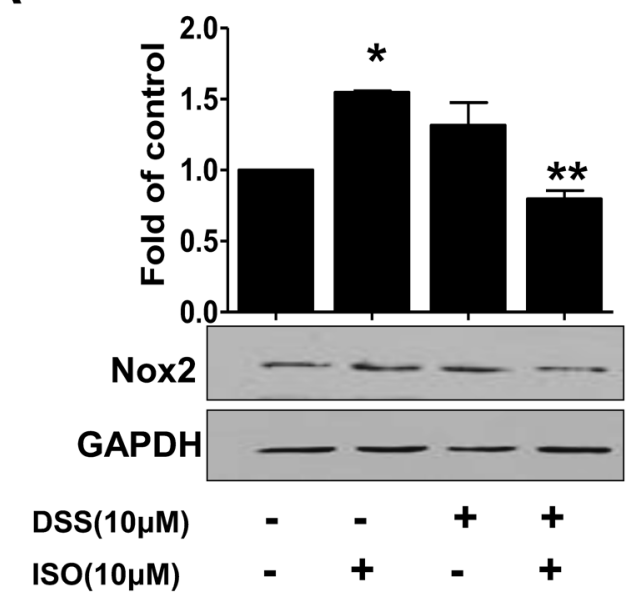

C

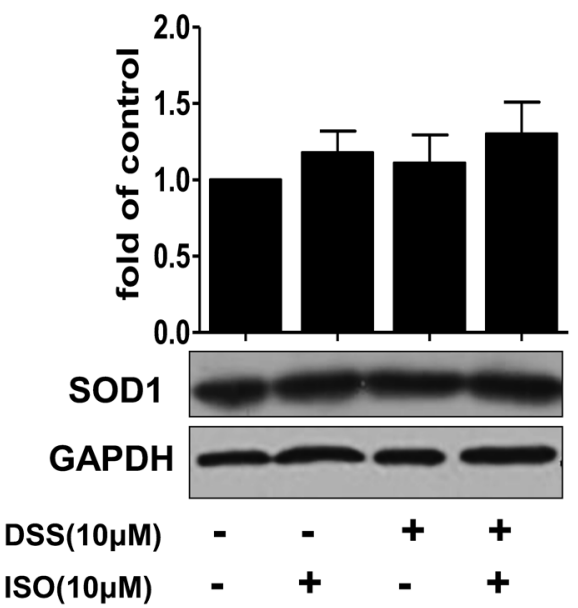

B

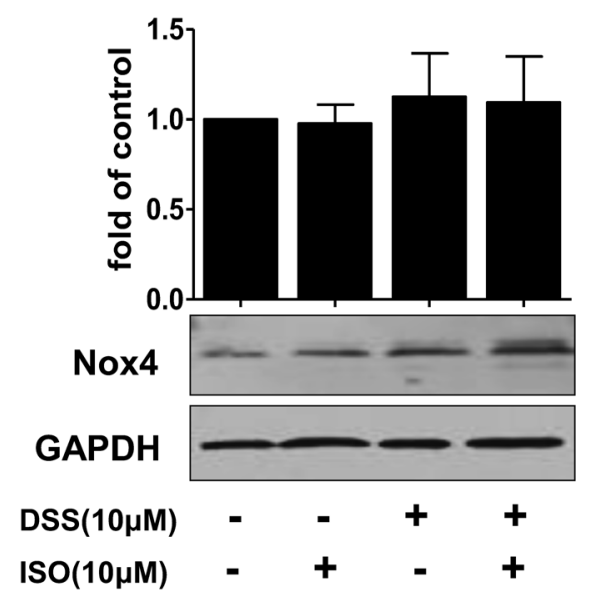

D

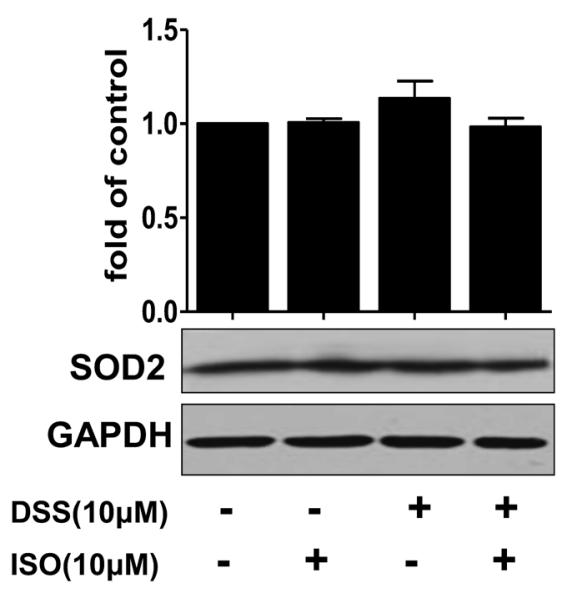

Fig. 6. Effect of DSS on ISO-Induced the Expression of NADPH Oxidase and Antioxidant Enzymes in CFs

Western blot analysis and quantification of the effect of DSS on ISO induced the expression of Nox2 (A), Nox4 (B), SOD1 (C) and SOD2 (D). * $p<0.01 v s$. con; $* * p<0.01$ vs. ISO. $N=3$. 


\section{DISCUSSION}

Excessive CF proliferation and the consequent increased collagen synthesis contribute significantly to cardiac fibrosis and inevitably lead to heart failure. Early intervention in the cardiac fibrogenesis process could slow down and prevent the progression of fibrosis. Although DSS has therapeutic effects on fibrosis of several organs, such as liver, ${ }^{26-28)}$ lung and kidney, its effect on cardiac fibrosis remained unclear. We investigated the effect of DSS on $\beta$-AR-mediated cardiac fibrosis and the involved signaling transduction. DSS inhibited CF proliferation and collagen I synthesis induced by ISO, a selective $\beta$-AR agonist; p38 MAPK phosphorylation, which mediates ISO-induced cardiac fibrosis, was negatively regulated in this process. The negative regulation depended on the inhibition of ISO-induced ROS production. DSS inhibits $\beta$-AR-mediated cardiac fibrosis by negatively regulating ROS-p38 MAPK signaling.

p38 MAPK is a class of evolutionarily conserved serine/ threonine MAPKs that link extracellular signals to the intracellular machinery to regulate multiple cellular processes including proliferation, differentiation, and inflammation. The MAPKs cascade can be activated by various receptors, including G-protein-coupled receptors by activating protein kinases $\mathrm{A}$ and $\mathrm{C}$ and can regulate cell proliferation and collagen synthesis. In particular, p38 activation is involved in CF proliferation as well as fibrosis of various organs. F2-Isoprostanes promote liver fibrosis by activating p38 MAPKs. ${ }^{29)}$ Inhibition of p38 MAPK can attenuate renal and lung fibrosis. ${ }^{23,30,31)}$ In addition, p38 MAPK plays an important role in CF proliferation. ${ }^{32,33)}$ In this study, CFs showed a significant increase in p38 phosphorylation when treated with ISO, as compared with untreated controls. Both the p38 inhibitor SB202190 and DSS significantly reduced p38 phosphorylation and CF proliferation. Therefore, p38 signaling is necessary for ISO-stimulated CF proliferation.

ROS is a major cause of cardiac diseases, including cardiac fibrosis and hypertrophy. It is generally considered the mechanism of cardiac injury in that ROS generation causes mitochondrial damage and alters gene expression in myocytes and fibroblasts. ${ }^{34)}$ Anti-oxidation can prevent adverse cardiovascular events. Indeed, drugs such as DSS play an anti-fibrosis role by reducing oxidant levels. ${ }^{35)}$ Interestingly, we showed that DSS can dose-dependently inhibit ISO-induced ROS production.

Imbalance between ROS production and elimination results in increased ROS accumulation. SOD is a class of enzymes that catalyze the dismutation of superoxide into oxygen and hydrogen peroxide. As such, it's an important antioxidant defense in nearly all cells exposed to oxygen. Our results showed that DSS had no effect on SOD1 and SOD2 protein expression in CFs, suggesting DSS decreased ISO-induced through inhibiting ROS production. ROS are produced in tracellularly through multiple mechanisms and depending on the cell and tissue types. The major source of ROS generated in the cardiovascular system is the NOX family of enzymes. In the present study, we found DSS inhibted ISO-induced ROS production depended on NOX2. NOX2 is expressed in vascular, cardiac, renal, and neural cells and localizes to intracellular and plasma membranes. Pathological increases in NOX2 contribute to oxidative injury, vascular and cardiac damage.
Consistent with our results, NOX2 has been shown to be important roles in models of cardiac remolding. ${ }^{36,37)}$

There are discrepancies in the relationship between ROS and p38 MAPK pathways. ROS was reported as the upstream signal molecule of p38 MAPK, directly regulating the phosphorylation of $\mathrm{p} 38$, and ultimately affecting biological effect. ${ }^{38-41)}$ However, some studies demonstrated contrary views. $^{42-44)}$ The different regulation networks may cause varied biological effects in diverse cells and organisms. To elucidate the specific relationship between ROS and p38, we checked the effect of NAC and SB202190, selective inhibitors of ROS and p38, respectively. NAC almost completely inhibited the phosphorylation of p38, but SB202190 was unable to suppress ISO-induced ROS production in CFs. Thus, p38 may be the downstream substrate of ROS. DSS inhibited CF proliferation induced by ISO by inhibiting ROS-p38 MAPK signaling.

In conclusion, our study indicates that DSS prevents CF proliferation and type I collagen synthesis induced by ISO by inhibiting ROS-p38 MAPK signaling. These findings will be beneficial to further understand the protective molecular mechanisms of DSS in the heart and provide potential drug targets for the treatment of heart diseases.

Acknowledgments This work was supported by Grants from the National Basic Research Program of China (No. 2011CB503903), the National Natural Science Foundation of China (Nos. 81030001, 81070078 and 81270157), Beijing Municipal Natural Science Foundation (No. 7102158), and Changjiang Scholars and Innovative Research Team in University on 2011 (No. IRT1174).

\section{REFERENCES}

1) Minnaard-Huiban M, Emmen JM, Roumen L, Beugels IP, Cohuet GM, van Essen H, Ruijters E, Pieterse K, Hilbers PA, Ottenheijm HC, Plate R, de Gooyer ME, Smits JF, Hermans JJ. Fadrozole reverses cardiac fibrosis in spontaneously hypertensive heart failure rats: discordant enantioselectivity versus reduction of plasma aldosterone. Endocrinology, 149, 28-31 (2008).

2) Tao H, Shi KH, Yang JJ, Huang C, Liu LP, Li J. Epigenetic regulation of cardiac fibrosis. Cell. Signal., 25, 1932-1938 (2013).

3) Ma F, Li Y, Jia L, Han Y, Cheng J, Li H, Qi Y, Du J. Macrophagestimulated cardiac fibroblast production of IL-6 is essential for TGF beta/Smad activation and cardiac fibrosis induced by angiotensin II. PLoS ONE, 7, e35144 (2012).

4) Koshman YE, Patel N, Chu M, Iyengar R, Kim T, Ersahin C, Lewis W, Heroux A, Samarel AM. Regulation of connective tissue growth factor gene expression and fibrosis in human heart failure. J. Card. Fail., 19, 283-294 (2013).

5) Sirish P, Li N, Liu JY, Lee KS, Hwang SH, Qiu H, Zhao C, Ma SM, Lopez JE, Hammock BD, Chiamvimonvat N. Unique mechanistic insights into the beneficial effects of soluble epoxide hydrolase inhibitors in the prevention of cardiac fibrosis. Proc. Natl. Acad. Sci. U.S.A., 110, 5618-5623 (2013).

6) Machackova J, Sanganalmath SK, Barta J, Dhalla KS, Dhalla NS. Amelioration of cardiac remodeling in congestive heart failure by beta-adrenoceptor blockade is associated with depression in sympathetic activity. Cardiovasc. Toxicol., 10, 9-16 (2010).

7) Zhao M, Fajardo G, Urashima T, Spin JM, Poorfarahani S, Rajagopalan V, Huynh D, Connolly A, Quertermous T, Bernstein D. Cardiac pressure overload hypertrophy is differentially regulated by beta-adrenergic receptor subtypes. Am. J. Physiol. Heart Circ. 
Physiol., 301, H1461-H1470 (2011).

8) Bisognano JD, Weinberger HD, Bohlmeyer TJ, Pende A, Raynolds MV, Sastravaha A, Roden R, Asano K, Blaxall BC, Wu SC, Communal C, Singh K, Colucci W, Bristow MR, Port DJ. Myocardialdirected overexpression of the human beta(1)-adrenergic receptor in transgenic mice. J. Mol. Cell. Cardiol., 32, 817-830 (2000).

9) Ayala P, Montenegro J, Vivar R, Letelier A, Urroz PA, Copaja M, Pivet D, Humeres C, Troncoso R, Vicencio JM, Lavandero S, DíazAraya G. Attenuation of endoplasmic reticulum stress using the chemical chaperone 4-phenylbutyric acid prevents cardiac fibrosis induced by isoproterenol. Exp. Mol. Pathol., 92, 97-104 (2012).

10) Zhang YG, Li YG, Liu BG, Wei RH, Wang DM, Tan XR, Bu DF, Pang YZ, Tang CS. Urotensin II accelerates cardiac fibrosis and hypertrophy of rats induced by isoproterenol. Acta Pharmacol. Sin., 28, 36-43 (2007).

11) Essick EE, Ouchi N, Wilson RM, Ohashi K, Ghobrial J, Shibata R, Pimentel DR, Sam F. Adiponectin mediates cardioprotection in oxidative stress-induced cardiac myocyte remodeling. Am. J. Physiol. Heart Circ. Physiol., 301, H984-H993 (2011).

12) Di Lisa F, Kaludercic N, Paolocci N. beta(2)-Adrenoceptors, NADPH oxidase, ROS and p38 MAPK: another 'radical' road to heart failure? Br. J. Pharmacol., 162, 1009-1011 (2011).

13) Chen C, Du J, Feng W, Song Y, Lu Z, Xu M, Li Z, Zhang Y. betaAdrenergic receptors stimulate interleukin-6 production through Epac-dependent activation of PKCdelta/p38 MAPK signalling in neonatal mouse cardiac fibroblasts. Br. J. Pharmacol., 166, 676-688 (2012).

14) Yin Y, Guan Y, Duan J, Wei G, Zhu Y, Quan W, Guo C, Zhou D, Wang Y, Xi M, Wen A. Cardioprotective effect of Danshensu against myocardial ischemia/reperfusion injury and inhibits apoptosis of $\mathrm{H} 9 \mathrm{c} 2$ cardiomyocytes via Akt and ERK1/2 phosphorylation. Eur. J. Pharmacol., 699, 219-226 (2013).

15) Pan LL, Liu XH, Jia YL, Wu D, Xiong QH, Gong QH, Wang Y, Zhu YZ. A novel compound derived from Danshensu inhibits apoptosis via upregulation of heme oxygenase-1 expression in SHSY5Y cells. Biochim. Biophys. Acta, 1830, 2861-2871 (2013).

16) Li H, Xie YH, Yang Q, Wang SW, Zhang BL, Wang JB, Cao W, Bi LL, Sun JY, Miao S, Hu J, Zhou XX, Qiu PC. Cardioprotective effect of paeonol and Danshensu combination on isoproterenolinduced myocardial injury in rats. PLoS ONE, 7, e48872 (2012).

17) Colombo F, Noel J, Mayers P, Mercier I, Calderone A. beta-Adrenergic stimulation of rat cardiac fibroblasts promotes protein synthesis via the activation of phosphatidylinositol 3-kinase. J. Mol. Cell. Cardiol., 33, 1091-1106 (2001)

18) Zhang Y, Wang Y, Liu Y, Wang N, Qi Y, Du J. Kruppel-like factor 4 transcriptionally regulates TGF-betal and contributes to cardiac myofibroblast differentiation. PLoS ONE, 8, e63424 (2013).

19) Shim JH, Park JY, Lee MG, Kang HH, Lee TR, Shin DW. Human dermal stem/progenitor cell-derived conditioned medium ameliorates ultraviolet a-induced damage of normal human dermal fibroblasts. PLoS ONE, 8, e67604 (2013).

20) Tian XY, Yung LH, Wong WT, Liu J, Leung FP, Liu L, Chen Y, Kong SK, Kwan KM, Ng SM, Lai PB, Yung LM, Yao X, Huang Y. Bone morphogenic protein-4 induces endothelial cell apoptosis through oxidative stress-dependent p38 MAPK and JNK pathway. J. Mol. Cell. Cardiol., 52, 237-244 (2012).

21) Li Z, Liu N, Zhang LS, Gong K, Cai Y, Gao W, Liu Z, Liu S, Han Q, Zhang Y. Proteomic profiling reveals comprehensive insights into adrenergic receptor-mediated hypertrophy in neonatal rat cardiomyocytes. Proteomics Clin. Appl., 3, 1407-1421 (2009).

22) Chen LX, Yang K, Sun M, Chen Q, Wang ZH, Hu GY, Tao LJ. Fluorofenidone inhibits transforming growth factor-betal-induced cardiac myofibroblast differentiation. Pharmazie, 67, 452-456 (2012).

23) Cowley EA. Isoprostane-mediated secretion from human airway epithelial cells. Mol. Pharmacol., 64, 298-307 (2003).
24) Okano K, Hibi A, Miyaoka T, Inoue $T$, Sugimoto $H$, Tsuchiya $K$, Akiba T, Nitta K. Inhibitory effects of the transcription factor Ets-1 on the expression of type I collagen in TGF-beta1-stimulated renal epithelial cells. Mol. Cell. Biochem., 369, 247-254 (2012).

25) Lu F, Xing J, Zhang X, Dong S, Zhao Y, Wang L, Li H, Yang F, Xu C, Zhang W. Exogenous hydrogen sulfide prevents cardiomyocyte apoptosis from cardiac hypertrophy induced by isoproterenol. $\mathrm{Mol}$. Cell. Biochem., 381, 41-50 (2013).

26) Zheng YY, Dai LL, Wang WB, Jia LP, Zhou X. Effect and mechanism of Tanshensu on fibrotic rats. Zhonghua Gan Zang Bing Za Zhi, 11, 288-290 (2003).

27) Li X, Peng XD, Zhang WL, Dai LL. Inhibiting effects of denshensu, baicalin, astragalus and Panax notoginseng saponins on hepatic fibrosis and their possible mechanisms. Zhonghua Gan Zang Bing Za Zhi, 16, 193-197 (2008).

28) Liang XL, Yuan JY. Effect of Chinese herbal compound on liver fibrosis in rabbits with schistosomiasis by B-ultrasound. Asian Pacific Journal of Tropical Medicine, 6, 658-662 (2013).

29) Acquaviva A, Vecchio D, Arezzini B, Comporti M, Gardi C. Signaling pathways involved in isoprostane-mediated fibrogenic effects in rat hepatic stellate cells. Free Radic. Biol. Med., 65, 201-207 (2013).

30) Wang D, Warner GM, Yin P, Knudsen BE, Cheng J, Butters KA, Lien KR, Gray CE, Garovic VD, Lerman LO, Textor SC, Nath KA, Simari RD, Grande JP. Inhibition of p38 MAPK attenuates renal atrophy and fibrosis in a murine renal artery stenosis model. $\mathrm{Am} . J$. Physiol. Renal Physiol., 304, F938-F947 (2013).

31) Xu X, Wan X, Geng J, Li F, Wang C, Dai H. Kinase inhibitors fail to induce mesenchymal-epithelial transition in fibroblasts from fibrotic lung tissue. Int. J. Mol. Med., 32, 430-438 (2013).

32) Chen LX, Yang K, Sun M, Chen Q, Wang ZH, Hu GY, Tao LJ. Fluorofenidone inhibits transforming growth factor-betal-induced cardiac myofibroblast differentiation. Pharmazie, 67, 452-456 (2012).

33) Wang LP, Wang Y, Zhao LM, Li GR, Deng XL. Angiotensin II upregulates $\mathrm{K}(\mathrm{Ca}) 3.1$ channels and stimulates cell proliferation in rat cardiac fibroblasts. Biochem. Pharmacol., 85, 1486-1494 (2013).

34) Schunke KJ, Coyle L, Merrill GF, Denhardt DT. Acetaminophen attenuates doxorubicin-induced cardiac fibrosis via osteopontin and GATA4 regulation: Reduction of oxidant levels. J. Cell. Physiol., 228, 2006-2014 (2013).

35) Yao Z, Hu W, Yin S, Huang Z, Zhu Q, Chen J, Zang Y, Dong L, Zhang J. 3,3'-Diindolymethane ameliorates adriamycin-induced cardiac fibrosis via activation of a BRCA1-dependent anti-oxidant pathway. Pharmacol. Res., 70, 139-146 (2013).

36) Nabeebaccus A, Zhang M, Shah AM. NADPH oxidases and cardiac remodelling. Heart Fail. Rev., 16, 5-12 (2011).

37) Cave A. Selective targeting of NADPH oxidase for cardiovascular protection. Curr. Opin. Pharmacol., 9, 208-213 (2009).

38) Liu JM, Pan F, Li L, Liu QR, Chen Y, Xiong XX, Cheng K, Yu SB, Shi Z, Yu AC, Chen XQ. Piperlongumine selectively kills glioblastoma multiforme cells via reactive oxygen species accumulation dependent JNK and p38 activation. Biochem. Biophys. Res. Commun., 437, 87-93 (2013).

39) Sánchez-Fidalgo S, de Silva MS, Cárdeno A, Aparicio-Soto M, Salvador MJ, Frankland Sawaya AC, Souza-Brito AR, de la Lastra CA. Abarema cochliacarpos reduces LPS-induced inflammatory response in murine peritoneal macrophages regulating ROS-MAPK signal pathway. J. Ethnopharmacol., 149, 140-147 (2013).

40) Cai W, Shi GX, Andres DA. Putting the Rit in cellular resistance: Rit, p38 MAPK and oxidative stress. Commun. Integr. Biol., 6, e22297 (2013).

41) Shi L, Yu X, Yang H, Wu X. Advanced glycation end products induce human corneal epithelial cells apoptosis through generation of reactive oxygen species and activation of JNK and p38 MAPK pathways. PLoS ONE, 8, e66781 (2013). 
42) Park WH. The effect of MAPK inhibitors and ROS modulators on cell growth and death of $\mathrm{H}_{2} \mathrm{O}_{2}$-treated HeLa cells. Mol. Med. Rep., 89, 557-564 (2013).

43) Su X, Wang P, Wang X, Guo L, Li S, Liu Q. Involvement of MAPK activation and ROS generation in human leukemia U937 cells undergoing apoptosis in response to sonodynamic therapy. Int. J.
Radiat. Biol., 89, 915-927 (2013).

44) Wang ZS, Luo P, Dai SH, Liu ZB, Zheng XR, Chen T. Salvianolic acid B induces apoptosis in human glioma U87 cells through p38-mediated ROS generation. Cell. Mol. Neurobiol., 33, 921-928 (2013). 